\title{
LET Monitoring Using Liquid Ionization Chambers
}

\author{
Sara Tegami', Stephen D. Bello², Shuang Luan ${ }^{3}$, Andrea Mairani ${ }^{4,5}$, Katia Parodi ${ }^{4,6}$, \\ Michael H. Holzscheiter ${ }^{1,2 *}$
}

\author{
${ }^{1}$ Max Planck Institute for Nuclear Physics, Heidelberg, Germany \\ ${ }^{2}$ Dept. of Physics \& Astronomy, University of New Mexico, Albuquerque, NM, USA \\ ${ }^{3}$ Dept. of Computer Science, University of New Mexico, Albuquerque, NM, USA \\ ${ }^{4}$ Heidelberg Ion Beam Therapy Center, Heidelberg University Hospital, Heidelberg, Germany \\ ${ }^{5}$ Medical Physics Unit, CNAO Foundation, Pavia, Italy \\ ${ }^{6}$ Dept. of Medical Physics, Ludwig-Maximilians-Universität München, Munich, Germany \\ Email: *michael.holzscheiter@gmail.com
}

How to cite this paper: Tegami, S., Bello, S.D., Luan, S., Mairani, A., Parodi, K. and Holzscheiter, M.H. (2017) LET Monitoring Using Liquid Ionization Chambers. International Journal of Medical Physics, Clinical Engineering and Radiation Oncology, 6, 197-207.

https://doi.org/10.4236/ijmpcero.2017.62018

Received: March 14, 2017

Accepted: May 23, 2017

Published: May 26, 2017

Copyright $(2017$ by authors and Scientific Research Publishing Inc. This work is licensed under the Creative Commons Attribution International License (CC BY 4.0).

http://creativecommons.org/licenses/by/4.0/

\begin{abstract}
Relative biological effectiveness (RBE) is an important quantity in planning particle beam cancer therapy. In general, the RBE describes the biological effectiveness of a given primary beam with respect to a reference photon irradiation. RBE varies not only for different primary beams but also with depth in the target for a given beam modality. It is not a quantity that easily lends itself to measurements or computation as it depends on many biological and physical quantities. Numerous experiments in vitro using various cell lines and irradiation modalities have shown that a general relationship between RBE and the physical quantity Linear Energy Transfer (LET) exists. Several groups have proposed including LET in the radiation therapy treatment planning instead of the more complicated and elusive RBE. It has been shown that LET is an important quantity to consider in treating radio-resistant tumors. The concept of LET painting has been proposed with the goal of improving tumor control probability (TCP) for hypoxic tumors by focusing high LET radiation on the hypoxic region of the tumor while restricting the surrounding normal tissue to low LET radiation. In order to properly incorporate LET in clinical treatment, it is important to be able to experimentally measure and verify LET distribution. We propose a novel method for measuring LET using a dual chamber methodology exploiting the difference in the observed recombination between air filled ionization chambers (IC) and liquid filled ionization chambers (LIC). The resulting difference in the measured signals will be used to directly extract the relative LET of an actual treatment beam in real time. This paper describes our initial studies of this method, presents preliminary results, and discusses further improvements toward a practical real-time LET measuring device.
\end{abstract}




\section{Keywords}

Linear Energy Transfer, Relative Biological Effectiveness, Liquid Ionization Chambers

\section{Introduction}

The use of hadrons (protons or heavy ions) in radiation cancer therapy offers several advantages over traditional X-ray, gamma, and electron beams. Heavy charged particles exhibit a unique depth dose profile with low dose at the entrance of the patient, a distinct increase in the dose at the end of the range (i.e. Bragg peak) and minimal dose delivered beyond the Bragg peak. This allows hadrons to deliver a highly conformal physical dose (i.e. energy deposited per unit mass) to deep-seated tumors with better sparing of nearby normal and critical structures than more conventional megavoltage photon beams. These physical advantages make hadron therapy attractive for modern cancer radiation therapy.

Additional research has shown that heavy ions are more effective at killing cells because they create denser ionization events along the particle track, which causes more irreparable damage to DNA than photons. This is described by the quantity of Relative Biological Effectiveness (RBE) - the ratio of the physical dose using photons to the physical dose needed for a given beam modality (e.g. heavy charged particles) to produce the same biological effect, for example $10 \%$ cell survival. Note that it has been widely recognized that even proton beams do not have a constant biological effectiveness along the beam path and that the enhanced biological effectiveness near the end of range should no longer be ignored for cases involving tumors in proximity to critical structures [1] [2].

$\mathrm{RBE}$ is a complex quantity dependent upon many different physical and biological parameters. Therefore, it may be considered unsuitable for treatment planning of complicated cancer incidences [3]. The physical quantity that is closely related to RBE and can be used to characterize biological damage is the LET-the mean energy locally imparted along the beam track to the medium by a particle. Numerous research groups have demonstrated a general relationship between the RBE and LET for both in vitro and in vivo cell lines. Figure 1 is taken from a recent survey [4] and shows a linear increase of RBE with LET up to a broad maximum where the effective RBE saturates and drops due to the "overkill" effect. The data represents not only different ion beams but also many independent experiments performed by various groups, which explains the scatter of the data points. For details on these measurements, please consult reference 4 .

From Figure 1, we observe that LET is a good surrogate for RBE between 10 $200 \mathrm{keV} / \mu \mathrm{m}$, which covers the clinical range for treatment planning, verification, and quality assurance in treatments using protons and carbon ions. This relationship between LET and RBE has led to proposals for optimizing treatment scenarios especially for radio-resistant and hypoxic tumors using the technique 


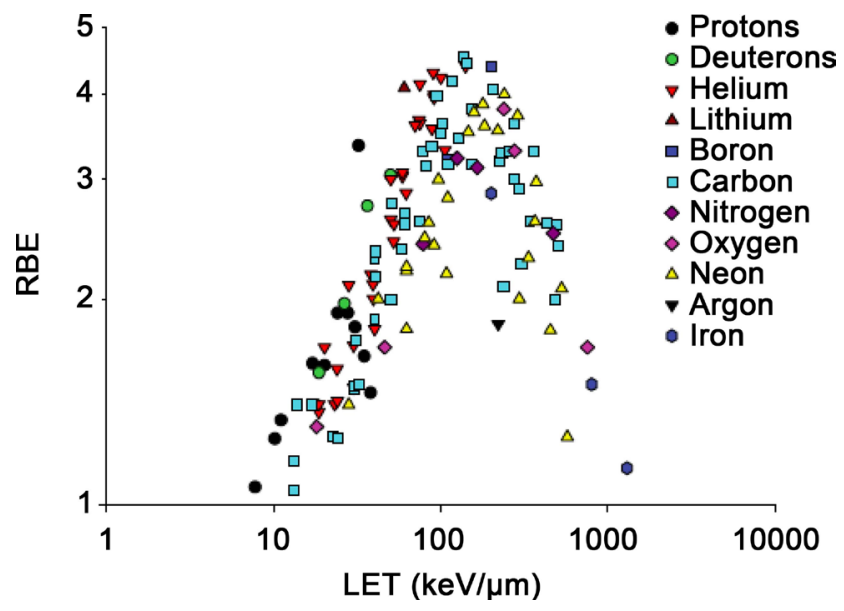

Figure 1. Relationship between RBE and LET for a multitude of heavy charged ions from protons to iron (from [4]).

of LET-painting [5] [6] where sub-volumes of the tumor are treated with highLET beams. This method could benefit significantly in its development and clinical use from the availability of a device to monitor LET in-situ during treatment.

In this paper, we will discuss a proposal for an instrument capable of monitoring LET in real time based on a dual ionization chamber method. We believe the device will ultimately enable (1) verification of hadron therapy treatment plans in real time and (2) "biological dose" optimization using LET as a physical surrogate for RBE.

\section{Preliminaries}

The detectors most widely used in radiotherapy are ionization chambers composed of two electrodes separated by an active medium consisting either of a gas or liquid. A radiotherapy beam passing through the chamber will ionize atoms and molecules in the active medium. The resulting positive or negative charge carriers are collected at the electrodes by applying an appropriate electric field between them. Due to processes of charge transfer, electron capture, and recombination, the quantity of charge carriers collected at the electrodes is not identical to the number of charges generated in the active medium, a signal loss we refer to as quenching. To obtain an accurate reading of the delivered dose, corrections for these charge losses must be applied.

One key advantage of a liquid-filled ionization chamber (LIC) over standard air filled chambers (IC) arises from the significantly higher mass density of the liquid, which allows the active volume to be smaller than that in ICs, resulting in a higher spatial resolution [7]. This makes LICs attractive for modern X-ray therapy (IMRT) and ion beam cancer therapy, where steep dose gradients between the target and the surrounding normal tissues may exist and must be carefully monitored. In addition to their higher spatial resolutions, LICs are water equivalent, exhibit long-term stability [8] and have small directional dependence [9].

However, the advantages arising from using LICs come with at least one sig- 
nificant drawback. Due to the higher density of the liquid medium, the distance between the ionization events is much shorter than in air-filled ionization chambers. This higher charge carrier density, together with low ion mobility in liquid medium, increases the probability of electron-ion recombination before the electrodes collect the charges, resulting in considerable quenching of the signal. Recombination effects taking place within ion chambers can be classified in two categories-Initial Recombination (IR) and General Recombination (GR).

Initial recombination occurs during the first tens of nanoseconds along the track of one individual primary particle. Theoretical description of initial recombination further distinguishes between geminate recombination, involving one electron and its mother ion [10], and columnar recombination, in which clusters formed along a single track expand due to ionic and electronic diffusion and overlap [11]. Initial recombination depends on the properties of the liquid medium, its temperature, and the external electric field $E$. It also depends upon the density of the ionization events along the primary particle's track and therefore on the linear energy transfer (LET) of the beam. IR is independent of the dose rate.

General recombination occurs between particles that are produced by different primary particles. The charge carriers that escape initial recombination can diffuse through the liquid medium, allowing electrons and ions originating from different primary particles to interact and undergo recombination. General recombination therefore depends, in addition to the liquid properties and the applied electric field, on the dose rate. It does not, however, depend on LET.

\section{Materials and Methods}

From the above discussion on initial and general recombination in LICs it becomes obvious that if one can determine the signal quenching due to the LET-dependent initial recombination in a LIC, then one can extract the LET of the beam. To achieve this, one will need to (1) suppress the signal quenching due to general combination and (2) determine the total number of charges before quenching.

To achieve (1) we note that general recombination in the LIC can be suppressed by a strong electric field [12]. To achieve (2) we note that due to the lower mass density of the medium in air-filled ionization chambers (ICs), both initial and general recombination can be considered negligible in comparison to the signal quenching observed in LIC's at conditions typically encountered in therapeutic settings. This implies that if one places an IC and a LIC at identical position, the IC reading would represent the normalized amount of charge released before recombination in the LIC reduces the signal. Specifically, we propose to measure LET by placing an IC and a LIC at the same location in a medium (e.g. water). Let $\mathrm{Q}_{\mathrm{IC}}$ and $\mathrm{Q}_{\mathrm{LIC}}$ be their respective charges collected. Assuming initial and general recombination in the IC can be ignored, and general recombination in the LIC is suppressed by the applied electric field, then the ratio 
$\mathrm{Q}_{I C} / \mathrm{Q}_{\mathrm{IIC}}$, which we define as the recombination index or quenching factor, is directly dependent on the LET.

To verify this idea, we have performed initial studies on differences in quenching by comparing the signals between a liquid-filled ionization and a commercial air-filled ionization chamber along the path of a clinical hadron beam entering a water phantom [13] [14].

\section{Experimental set-up:}

The experiments described here were performed at the Heidelberg Ion Beam Therapy Center (HIT). The experimental set-up (see Figure 2) consisted of a PTW phantom water tank, two air-filled ionization chambers (PTW Bragg peak chamber type 34,070) and a $2 \mathrm{D}$ liquid ionization chamber array comprised of 16 $\times 8$ channels, $1.8 \times 1.8 \mathrm{~mm}$ each, at a pitch of $2 \mathrm{~mm}$, for a total sensitive area of $3.2 \times 1.6 \mathrm{~cm}^{2}$, filled with isooctane $\left(2,2,4-\right.$ Trimethylpentane, $\left.\mathrm{C}_{8} \mathrm{H}_{18}\right)$. (The $2 \mathrm{D}$ array was originally developed by Prof. F. Gomez's group at the Universidad de Santiago de Compostela (USC) for dynamic multileaf collimator (MLC) verification [15] [16].)

As shown in Figure 2, one air-filled chamber (IC1) was mounted at the front window of the phantom to monitor the incoming beam from spill to spill for normalization purposes. The actual measurement air chamber (IC2) was attached to the PMMA enclosure protecting the LIC array from exposure to water during the experimental sessions.

A typical measurement cycle consisted of recording the signal of the IC and the LIC while moving the assembly in $1 \mathrm{~cm}$ steps in the plateau region $(0-60$ $\mathrm{mm}$ ) and then in $1 \mathrm{~mm}$ steps until both the IC and LIC had passed the Bragg peak. The beam diameter at the entrance window for the phantom was chosen to be smaller than the active area of the LIC. However, due to lateral scattering of the beam in water this could not be guaranteed for the full depth covered by the measurements. Corrections for this effect were applied during the final data analysis using the actual beam profile at every given depth using the information provided by the pixilated matrix of the liquid ionization chamber. In first ap-

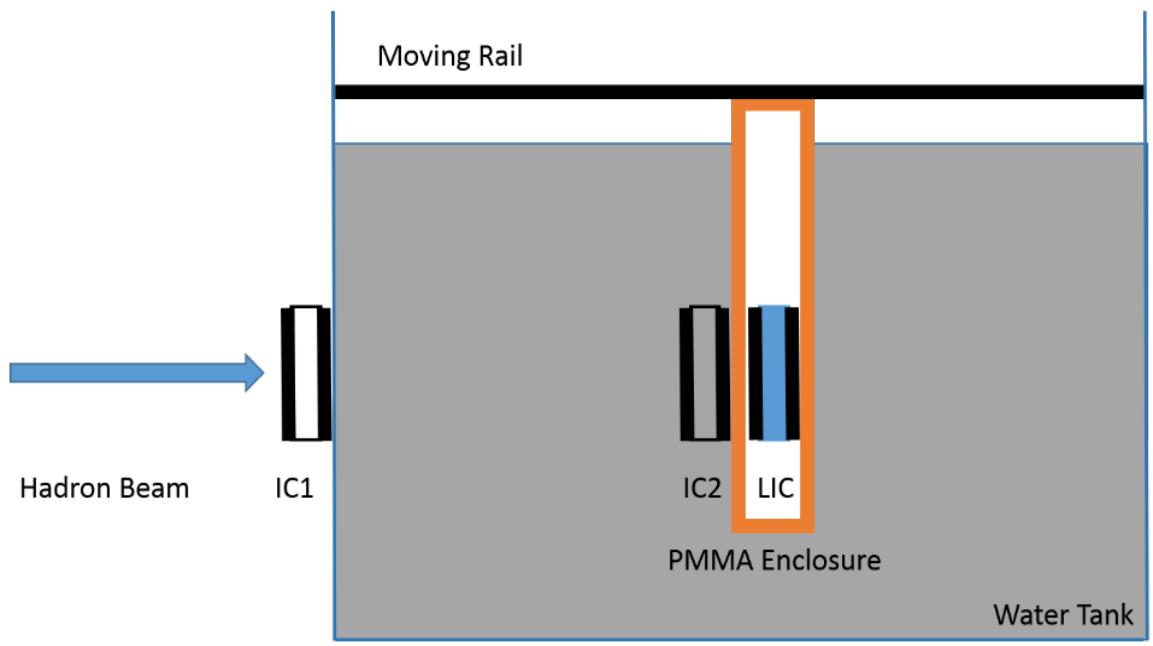

Figure 2. Schematic illustration of our experimental setup. 
proximation, the observed $2 \mathrm{D}$ radial profile in $\mathrm{x}$ and $\mathrm{y}$ direction was extended linearly beyond the boundary of the LIC and the signal lost due to the portion of the beam outside the active area of the LIC was estimated. Due to the physical separation between the IC and LIC, the recorded LIC depth dose curve had to be shifted with respect to the IC curve so that the measurements from the chambers coincide at identical depths. Unfortunately, the actual construction of the experimental set-up did not allow determining the distance between the active volumes of the two chambers at sufficient accuracy and the following two methods were used instead: (a) shifting the LIC curve to match the positions of the Bragg peaks of the IC and LIC curves and (b) shifting the LIC curve to match the 50\% distal fall off for the IC and LIC curves. Effects of strongly varying signal quenching near the end of range on position and shape of the Bragg peak were ignored in these preliminary analyses. An example of a typical data set using method (a) is shown in Figure 3.

\section{LET calculation:}

As stated previously, the ratio between IC and LIC signals depends on the LET. To extract the LET values in a practical application, one first needs to create a "lookup table" for the quenching ratio to the LET. To construct such tables, we calculate the LET values of the incoming beam including all details of the experimental set-up, using FLUKA [17] [18]. FLUKA is a particle transport code that includes all electromagnetic physical processes and hadronic interactions from few $\mathrm{keV}$ up to $10,000 \mathrm{TeV}$, and has been thoroughly benchmarked against other models and experiments specific to particle therapy [19] [20]. The

Dose response of IC and LIC

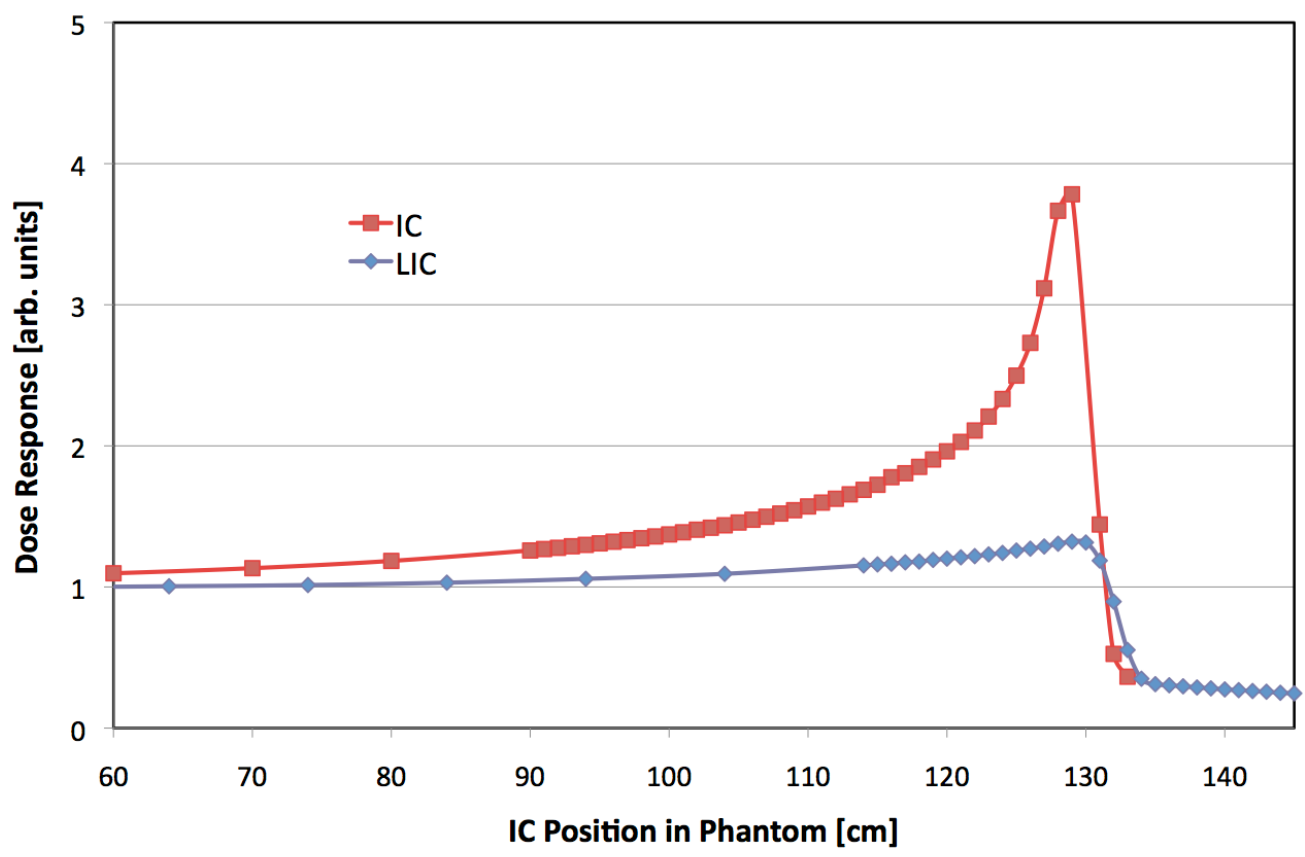

Figure 3. Depth dose distribution for a carbon ion beam as measured with an IC and a LIC. Quenching of the LIC signal due to initial recombination increases dramatically near the Bragg peak. The depth dose profile for the LIC was shifted to match the position of the Bragg peak. 
transport of charged particles is performed using a multiple Coulomb scattering algorithm [21] and the treatment of energy loss due to ionization is based on the first Born approximation. A complete description of FLUKA can be found at www.fluka.org, and details of the FLUKA modeling of the HIT beam-line can be found in [22] [23].

We used FLUKA to calculate the dose-averaged LET of the primary beam. A particle beam delivered from the accelerator will be modified through nuclear reactions in the target, creating a changing particle spectrum along the depth dose profile. This is especially pronounced for heavier ions, where various light fragments are produced that contribute to the dose. This demands using average LET values instead of pure LET values. For clinical applications, the relevant averaging scheme is the dose averaged $L E T^{D}$ :

$$
L E T^{D}=\int D(L) \mathrm{d} L / D_{\text {total }},
$$

where $D(L) \mathrm{d} L$ is the fraction of the total dose with the specific LET value $L$. The integral is normalized by the total dose $D_{\text {total }}$. LET $T^{D}$ cannot be directly scored in FLUKA and therefore a custom user routine was developed to perform the averaging over the particle-energy spectrum at each depth point.

This was achieved by customizing the user routine fluscw.f in order to weight on-the-fly the particle fluence spectrum in water $\phi_{\mathrm{w}, \mathrm{i}}(E)$ with its related squared stopping power $S_{w, i}^{2}(E)$, and sum it up over all the $i$-th components of the mixed radiation field at each depth in water. In a similar way, the total dose $D_{w}$ was scored in the same simulation run with the identical depth resolution:

$$
D_{w}=\sum_{i} \int \Phi_{w, i}(E)\left(S_{w, i}(E) / \rho_{w}\right) \mathrm{d} E
$$

The dose averaged $L E T^{D}$ for each depth was thus finally calculated at the end of the simulation as:

$$
L E T^{D}=\frac{\sum_{i} \int \frac{\Phi_{w, i}}{\rho_{w}}(E)\left(S_{w, i}(E)\right)^{2} \mathrm{~d} E}{D_{w}}
$$

Dose and the $L E T^{D}$ have been scored using $20 \mu \mathrm{m}$ bins in the direction of the beam.

\section{Results}

We define the ratio of the signal response from the IC and the signal from the LIC as the quenching factor or recombination index. The calculated values for $\mathrm{LET}^{\mathrm{D}}$ are then plotted against the measured recombination index. Figure 4 shows the LET $^{\mathrm{D}}$ distribution for a carbon beam with beam energy of 287.02 $\mathrm{MeV} / \mathrm{u}$. We observe a steady increase of the recombination index with increasing LET. The two curves represent the two different methods for matching the IC and LIC positions. These results agree well up to the end of the depth dose distribution $(\sim 1 \mathrm{~mm}$ before the Bragg peak) where the steep increase in LET leads to deviations between the two approaches even for differences in the relative IC and LIC positions on the order of less than $1 \mathrm{~mm}$ obtained using the 
Dose Averaged LET vs. Recombination Index

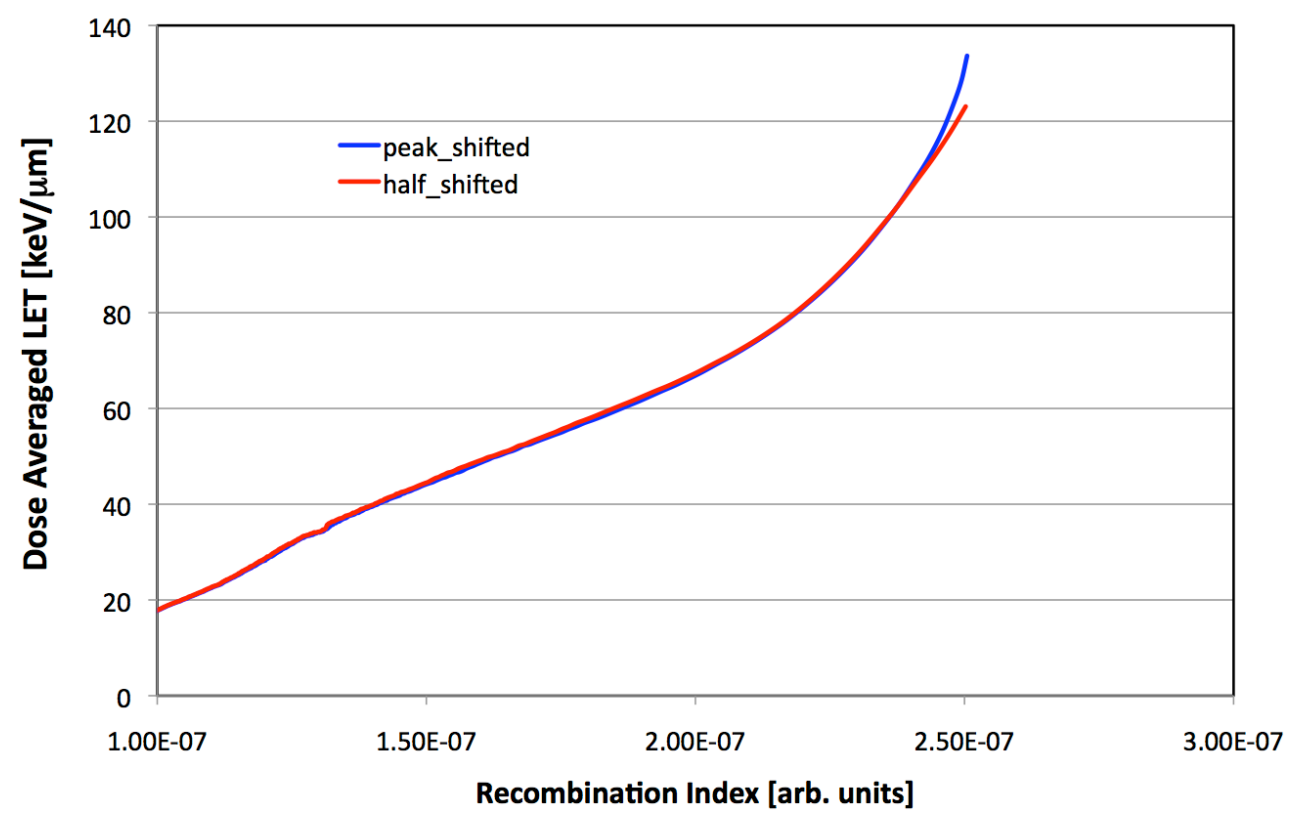

Figure 4. Calculated dose averaged LET vs. measured recombination index for an incoming carbon beam of $287.02 \mathrm{MeV} / \mathrm{u}$.

different methods. The maximum variation between the two methods is approximately $10 \mathrm{keV} / \mu \mathrm{m}$, which is less than $10 \%$ with respect to the maximum LET encountered.

The data used to generate Figure 4 have been corrected for the mismatch of the beam size and the size of the active area of the LIC, but no correction has been applied for general recombination effects. As previously mentioned, at the applied electric field strength general recombination is strongly suppressed. Any correction is negligible compared to the main source of error-the relative shift of the LIC curve to the IC curve for both measurements to represent identical depths in the target. We believe that the deviation from a straight line near the end of range is a consequence of the uncertainty in the determination of the relative position of IC2 and LIC. Similar behavior was observed by the authors of reference [24] who used a plastic scintillation detector (PSD) to study the dependence of the quenching correction factor (QCF) on LET. Here the optimal shift of the axial distance was found by postulating a linear dependency and then minimizing the RMS error to best fit of the data to a straight line. Significant changes in the curvature of the QCF vs. LET relation were found for variations of $0.2 \mathrm{~mm}$ or less in the shift, which is smaller than the accuracy with which this distance was known in our preliminary tests. Clearly the best method of settling the problem of relative position is not inferring it via an a priori postulate, but designing a dedicated dual chamber system where this quantity is known to high precision.

\section{Conclusion and Current Ongoing Efforts}

Our initial measurements have demonstrated a relationship between the recombination index (relative signal ratio obtained between an air-filled and liquid- 
filled ionization chamber) and the (calculated) dose-averaged LET, which is the quantity one would need for treatment planning calculations. We speculate that this should allow extracting the $L E T^{D}$ for an arbitrary incoming beam at any location of interest in the target by simply measuring the recombination index at that point, and then looking up the corresponding LET value using a pre-computed look-up table.

We are currently in the process of constructing a dedicated dual ionization chamber system, which is designed to overcome the uncertainties presented in the pilot studies. Specifically, we will use a monolithic system with a minimal and well-defined distance between the IC and LIC active volumes, separated only by a thin common electrode, to eliminate any uncertainties in relative position for the air and liquid volumes. Both chambers will also have identical active areas, eliminating the need for correcting the expansion of the beam as a function of depth.

Planned studies include irradiations using different therapeutic ion beams ( $p$, $\mathrm{C}, \mathrm{O}$ ) at different energies, using different means of energy modification (passive or active). The relative change in the quenching factor will allow detecting LET changes and thus can be used to verify planned LET enhancements along the beam path or due to overlapping beams of low and high LET components (LETpainting). We are hopeful that this will lead to a method to determine the absolute value of LET in an arbitrary beam at any position. LET averaging over a complex particle/energy spectrum could potentially lead to different quenching factors at identical values of $L E T^{D}$. These questions will be addressed with planned future measurements with our dual chamber system. The long-term goal is miniaturizing the system, allowing its use for in-vitro and in-vivo measurements with clinically relevant resolutions.

\section{Acknowledgements}

This research was supported in part by NIH R21 CA197325-02 and DPG WE3565-3. The corresponding author Michael Holzscheiter acknowledges the financial support by the Marie Curie Action PIIF-GA-2009-234814. We gratefully acknowledge the support and hospitality of Prof. O. Jäkel and the entire staff at the Heidelberg Ion Therapy Center. A special "Thank You" goes to Dr. Stefan Brons, who stayed with us through many night shifts supporting us. Prof. F. Gomez provided us with the Liquid Ionization Chamber array and introduced us to the intricacies of working with LICs, Prof. $\mathrm{H}$. Tölli provided us valuable advice in the understanding of recombination processes in ionization chambers.

\section{References}

[1] Wouters, B.G., Lam, G.K.Y., Oelfke, U., Gardey, K., Durand, R.E. and Skarsgard, L.D. (1996) Measurements of Relative Biological Effectiveness of the $70 \mathrm{MeV}$ Proton Beam at TRIUMF Using Chinese Hamster V79 Cells and the High-Precision Cell Sorter Assay. Radiation Research, 146, 159-170. https://doi.org/10.2307/3579588

[2] Paganetti, H. (2014) Relative Biological Effectiveness (RBE) Values for Proton Beam 
Therapy. Variations as a Function of Biological Endpoint, Dose, and Linear Energy Transfer. Physics in Medicine and Biology, 59, R419-R472. https://doi.org/10.1088/0031-9155/59/22/R419

[3] Lomax, T. (2016) Invited Talk at ICTR-PHE 2016, Geneva, Switzerland.

[4] Singers Sørensen, B., Overgaard, J. and Bassler, N. (2011) In Vitro RBE-LET Dependence for Multiple Particle Types. Acta Oncologica, 50, 757-762. https://doi.org/10.3109/0284186X.2011.582518

[5] Bassler, N., Jäkel, O., Søndergaard, S.S. and Petersen, J.B. (2010) Dose- and LETPainting with Particle Therapy. Acta Oncologica, 49, 1170-1176. https://doi.org/10.3109/0284186X.2010.510640

[6] Bassler, N., et al. (2014) LET-Painting Increases Tumour Control Probability in Hypoxic Tumours. Acta Oncologica, 53, 25-32. https://doi.org/10.3109/0284186X.2013.832835

[7] Wickman, G. (1974) A Liquid Ionization Chamber with High Spatial Resolution. Physics in Medicine and Biology, 19, 66-72. https://doi.org/10.1088/0031-9155/19/1/006

[8] Bahar-Gogani, J., Grindborg, J.E., Johansson, B.E. and Wickman, G. (2001) LongTerm Stability of Liquid Ionization Chambers with Regard to Their Qualification as Local Reference Dosimeters for Low Dose-Rate Absorbed Dose Measurements in Water. Physics in Medicine and Biology, 46, 729-740. https://doi.org/10.1088/0031-9155/46/3/309

[9] Dasu, A., Löfroth, P. and Wickman, G. (1998) Liquid Ionization Chamber Measurements of Dose Distributions in Small 6 MV Photon Beams. Physics in Medicine and Biology, 43, 21-36. https://doi.org/10.1088/0031-9155/43/1/002

[10] Onsager, L. (1938) Initial Recombination of Ions. Physical Review, 54, 554-557. https://doi.org/10.1103/PhysRev.54.554

[11] Jaffé, G. (1940) On the Theory of Recombination. Physical Review, 58, 968-976. https://doi.org/10.1103/PhysRev.58.968

[12] Johansson, B. and Wickman, G. (1997) General Collection Efficiency for Liquid Isooctane and Tetramethylsilane Used as Sensitive Media in a Parallel-Plate Ionization Chamber. Physics in Medicine and Biology, 42, 133-145. https://doi.org/10.1088/0031-9155/42/1/009

[13] Tegami, S. (2012) Liquid Ionization Chambers for Quality Assurance of High-Let Beams. International Conference on Translational Research-Physics for Health, ICTR-PHE 2012, Geneva.

[14] Tegami, S. (2013) LET Measurements with a Liquid Ionization Chamber. Ph.D. Thesis, University of Heidelberg, Heidelberg.

[15] Eberle, K., et al. (2003) First Tests of a Liquid Ionization Chamber to Monitor Intensity Modulated Radiation Beams. Physics in Medicine and Biology, 48, 35553564. https://doi.org/10.1088/0031-9155/48/21/007

[16] Pardo, J., et al. (2005) Development and Operation of a Pixel Segmented LiquidFilled Linear Array for Radiotherapy Quality Assurance. Physics in Medicine and Biology, 50, 1703-1716. https://doi.org/10.1088/0031-9155/50/8/006

[17] Ferrari, A., Sala, P.R., Fasso, A. and Ranft, J. (2005) FLUKA: A Multi-Particle Transport Code. CERN-2005-10 INFN/TC_05/11, SLAC-R-773.

[18] Böhlen, T., et al. (2014) The FLUKA Code: Developments and Challenges for High Energy and Medical Applications. Nuclear Data Sheets, 120, 211-214. https://doi.org/10.1016/j.nds.2014.07.049

[19] Mairani, A., Brons, S., Cerutti, F., Fassó, A., Ferrari, A., Krämer, M., Parodi, K., 
Scholz, M. and Sommerer, F. (2010) The FLUKA Monte Carlo Code Coupled with the Local Effect Model for Biological Calculations in Carbon Ion Therapy. Physics in Medicine and Biology, 55, 4273-4289.

https://doi.org/10.1088/0031-9155/55/15/006

[20] Böhlen, T.T., Cerutti, F., Dosanjh, M., Ferrari, A., Gudowska, I., Mairani, A. and Quesada, J.M. (2010) Benchmarking Nuclear Models of FLUKA and GEANT4 for Carbon Ion Therapy. Physics in Medicine and Biology, 55, 5833-5847.

https://doi.org/10.1088/0031-9155/55/19/014

[21] Ferrari, A., et al. (1992) An Improved Multiple Scattering Model for Charged Particle Transport. Nuclear Instruments and Methods, B71, 412-426. https://doi.org/10.1016/0168-583X(92)95359-Y

[22] Parodi, K., Mairani, A., Brons, S., Hasch, B.G., Sommerer, F., Naumann, J., Jäkel, O., Haberer, T. and Debus, J. (2012) Monte Carlo Simulations to Support Start-Up and Treatment Planning of Scanned Proton and Carbon Ion Therapy at a Synchrotron-Based Facility. Physics in Medicine and Biology, 57, 3759-3784. https://doi.org/10.1088/0031-9155/57/12/3759

[23] Parodi, K., Mairani, A. and Sommerer, F. (2013) Monte Carlo-Based Parametrization of the Lateral Dose Spread for Clinical Treatment Planning of Scanned Proton and Carbon Ion Beams. Journal of Radiation Research, 54, 191-196. https://doi.org/10.1093/jrr/rrt051

[24] Wang, L.L.W., Perles, L.A., Archambault, L., Sahoo, N., Mirkovic, D. and Beddar, S. (2012) Determination of the Quenching Correction Factors for Plastic Scintillation Detectors in Therapeutic High-Energy Proton Beams. Physics in Medicine and Biology, 57, 7767-7781. https://doi.org/10.1088/0031-9155/57/23/7767

Submit or recommend next manuscript to SCIRP and we will provide best service for you:

Accepting pre-submission inquiries through Email, Facebook, LinkedIn, Twitter, etc. A wide selection of journals (inclusive of 9 subjects, more than 200 journals)

Providing 24-hour high-quality service

User-friendly online submission system

Fair and swift peer-review system

Efficient typesetting and proofreading procedure

Display of the result of downloads and visits, as well as the number of cited articles

Maximum dissemination of your research work

Submit your manuscript at: http://papersubmission.scirp.org/

Or contact ijmpcero@scirp.org 